

И. И. Иванчик, Аналитическое представление уравнения состояния в классической статистической механике, ТМФ, 1996, том 108, номер 1, 135-158

DOI: https://doi.org/10.4213/tmf1183

Использование Общероссийского математического портала Math-Net.Ru подразумевает, что вы прочитали и согласны с пользовательским соглашением

http://www . mathnet.ru/rus/agreement

Параметры загрузки:

IP: 18.207 .199 .55

26 апреля 2023 г., 11:30:03 
ТЕОРЕТИЧЕСКАЯ

И МАТЕМАТИЧЕСКАЯ

ФИЗИКА

Том 108, № 1

июль, 1996

И.И. Иванчик

\title{
АНАЛИТИЧЕСКОЕ ПРЕДСТАВЛЕНИЕ УРАВНЕНИЯ СОСТОЯНИЯ В КЛАССИЧЕСКОЙ СТАТИСТИЧЕСКОЙ МЕХАНИКЕ
}

\begin{abstract}
Для системы классических частиц, взаимодействующих парными короткодействующими силами, построены аналитические представления модуля всестороннего сжатия в виде явно сходящихся рядов по обычной и дополняющей плотностям и в виде контурных интегралов. Аналогичные представления даны для уравнения состояния и удельного логарифма конфигурационного интеграла. Знания модуля всестороннего сжатия достаточно для нахождения всех представляющих интерес термодинамических величин и он, по-видимому, однозначен в окрестности фазового перехода. Построенный аппарат апробирован на точно решаемой модели - "вешестве Ван-дер-Ваальса" - модельном "веществе", для которого уравнение Ван-дер-Ваальса является точным уравнением состояния.
\end{abstract}

\section{1. ВВЕДЕНИЕ}

В статьях Калмыкова [1] конструктивно решена проблема асимптотической катастрофы обобщенных майеровских рядов, сформулированная в работах [2]. Тем самым устранено препятствие, затруднявшее эффективное развитие майеровского подхода [3] к равновесной статистической механике.

Цель настоящей статьи - продемонстрировать следствия для физики, вытекаюшие из математических результатов работ [1]. Рассматриваются подходы к теории уравнения состояния и фазового перехода пар-жидкость в равновесных системах классических частиц, взаимодействуюших парными силами короткого радиуса действия. Системы с кулоновским дальнодействием будут рассмотрены в дальнейшем.

Чисто математическим вопросам, связанным с проблемой асимптотической катастрофы майеровских рядов [2] и ее решением в статьях [1], посвяшен п.1 приложения к данной статье.

\section{2. ДВОЙСТВЕННОЕ ОПИСАНИЕ СИСТЕМЫ ЧАСТИЦ}

Кроме математического удобства (устранение асимптотической катастрофы), найденные в [1] представления обобшенных майеровских рядов удобны и с чисто физической точки зрения, поскольку позволяют явно учесть в теории важнейшее свойство классических частиц - наличие у них эффективного размера (обусловленного отталкиванием на близких расстояниях). Системы частиц, допускающие конструктивное введение 
конечного “объема частицы” $v_{a}$, назовем в данной работе "ван-дер-ваальсовыми системами”. Для ван-дер-ваальсовых систем возможно двойственное описание уравнения состояния - в терминах удельного объема (нормированного на $v_{a}$ )

$$
v=\frac{V}{N v_{a}}
$$

и дополнительное описание в терминах дополняющего объема $\frac{V}{N}-v_{a}$ (дополняющего объем частицы $v_{a}$ до удельного объема $\left.\frac{V}{N}\right)$, который после нормировки на $v_{a}$ записывается в виде

$$
w=v-1
$$

Двойственное описание уравнения состояния может быть полезно, поскольку имеющие физический смысл значения обычной плотности

$$
n=\frac{1}{v}
$$

заключены в интервале $0<n<1$, в то время как для дополняюшей плотности

$$
u=\frac{1}{w}
$$

физически осмысленные значения заполняют всю положительную полуось $0<u<\infty$. Обычная и дополняюшая плотности связаны тождеством

$$
\frac{1}{n}-\frac{1}{u}=1
$$

2.1. Объем частицы. Для экономии места в настоящей статье будем считать известными результаты Калмыкова [1] и введенные им понятия. Полученное в работах [1] выражение для плотности числа частищ $n_{s}=\frac{N}{V}$ эквивалентно следующему ряду Лорана для удельного объема $v_{s}=\frac{V}{N}$ :

$$
v_{s}(z)=\frac{1}{z}-\chi_{1}-\sum_{k=2}^{\infty} \chi_{k} z^{k-1},
$$

где $z$ - обычная активность [4], а $\chi_{k}=(k+1) a_{k+1}, k=1,2, \ldots$ (величины $a_{n}, n=$ $2,3, \ldots$, определены в [1]).

В статьях [1] фактически показано, что ряд для функции $v_{s}(z)$ сходится в некотором круге, следовательно, она является аналитической и можно рассмотреть вопрос об ее аналитическом продолжении на все $z>0$ и, в частности, вопрос об ее асимптотике при $z \rightarrow+\infty$.

Определим ван-дер-ваальсову систему как такую систему классических частиц, для которой выполняется асимптотическое условие

$$
\lim _{z \rightarrow+\infty} v_{s}(z)=v_{a}=\text { const }>0 .
$$

Класс ван-дер-ваальсовых систем не пуст, так как он содержит рассмотренное ниже модельное вещество Ван-дер-Ваальса (см. раздел 3). 
Для систем частиц из класса Ван-дер-Ваальса величину $v_{a}(2.7)$ будем трактовать как "объем частицы". Вводя безразмерную активность $\zeta$

$$
\zeta=z v_{a},
$$

получаем из (2.6) для удельного объема (2.1)

$$
v=\alpha(\zeta)=\frac{\alpha_{0}}{\zeta}+\sum_{k=1}^{\infty} \alpha_{k} \zeta^{k-1}
$$

где

$$
\alpha_{0}=1, \quad \alpha_{k}=-\frac{\chi_{k}}{v_{a}{ }^{k}}, \quad k=1,2, \ldots
$$

Для дополняющего объема согласно (2.2)

$$
w=\widetilde{\alpha}(\zeta)=\frac{\widetilde{\alpha}_{0}}{\zeta}+\sum_{k=1}^{\infty} \widetilde{\alpha}_{k} \zeta^{k-1}
$$

где

$$
\widetilde{\alpha}_{0}=1, \quad \widetilde{\alpha}_{1}=-1-\frac{\chi_{1}}{v_{a}}, \quad \widetilde{\alpha}_{k}=-\frac{\chi_{k}}{v_{a}^{k}}, \quad k=2,3, \ldots
$$

По определению

$$
\begin{aligned}
& \lim _{\zeta \rightarrow+\infty} \alpha(\zeta)=1 \\
& \lim _{\zeta \rightarrow+\infty} \widetilde{\alpha}(\zeta)=0
\end{aligned}
$$

Величины $v, w, \zeta$ ввиду их специфической нормировки будем назьвать соответственно, нормальным удельным обвемом, нормальным дополняющим обвемоми нормальной активностью. Нормальные параметры безразмерны.

2.2. Модуль всестороннего сжатия. Модуль всестороннего сжатия

$$
K=-V\left(\frac{\partial P}{\partial V}\right)_{T}
$$

допускает двойственное представление в виде аналитической функции обычной плотности $n$ (2.3) (вириальное представление) и аналитической функции дополняющей плотности $u$ (2.4) (дополнительное представление). Этим двум представлениям соответствует двойственное представление уравнения состояния системы частиц в дифференциальной форме.

Введем безразмерную величину $\varkappa$

$$
\varkappa=\beta K v_{a}
$$

где $v_{a}$ определено формулой (2.7), а

$$
\beta=\frac{1}{k T},
$$


и рассмотрим две аналитические функции $\psi(n)$ и $\widetilde{\psi}(u)$, выражаюшие $\varkappa$ соответственно в виде функции от обычной плотности $n(2.3)$

$$
\varkappa=n \psi(n) \quad \text { (вириальное представление) }
$$

и в виде функции от дополняюшей плотности $u(2.4)$ :

$$
\varkappa=u \widetilde{\psi}(u) \quad \text { (дополнительное представление). }
$$

Поскольку функции $\psi(n)$ и $\widetilde{\psi}(u)$ играют в дальнейшей аргументации заметную роль, введем для них специальные названия: о $\psi(n)$ будем говорить как о вириальном нормальном модуле, а о $\widetilde{\psi}(u)$ - как о дополнительном нормальном модуле.

Для построения вириального и дополнительного нормальных модулей нам потребуются функции, обратные функциям (2.9) и (2.11), выражающие нормальную активность $\zeta$ соответственно через нормальную плотность $n(2.3)$ и через дополняющую плотность $u(2.4)$, именно:

$$
\begin{array}{ll}
\zeta=\varphi(n) & \text { (вириальное представление) }, \\
\zeta=\widetilde{\varphi}(u) & \text { (дополнительное представление). }
\end{array}
$$

С их помощью получаем (см. п.2 приложения) для вириального нормального молуля

$$
\psi(n)=n \frac{d \ln \varphi(n)}{d n}
$$

и для дополнительного нормального модуля

$$
\widetilde{\psi}(u)=u \frac{d \ln \widetilde{\varphi}(u)}{d u}
$$

Конкретные выражения для $\psi(n)$ и $\widetilde{\psi}(u)$ рассматриваются ниже в п.2.3 и 2.4 .

Вводя вместо давления $P$ новую переменную $p$, имеюшую размерность энергии

$$
p=P v_{a},
$$

приходим к следуюшему двойственному представлению уравнения состояния в дифференциальной форме:

$$
\begin{aligned}
& \beta \frac{d p}{d n}=\psi(n) \quad(\text { вириальное представление }), \\
& \beta \frac{d p}{d u}=\frac{1}{1+u} \widetilde{\psi}(u) \quad \text { (дополнительное представление) } .
\end{aligned}
$$


2.3. Представления уравнения состояния рядами. Функции $\zeta=\varphi(n)(2.20)$ и $\zeta=\widetilde{\varphi}(u)(2.21)$, обратные функциям $v=\alpha(\zeta)(2.9)$ и $w=\widetilde{\alpha}(\zeta)(2.11)$, построены в п.3 приложения, а в п.4 приложения дан вывод явных выражений для функций $\psi(n)(2.22)$ и $\widetilde{\psi}(u)(2.23)$.

Для вириального нормального модуля $\psi(n)$ имеем

$$
\psi(n)=\sum_{k=0}^{\infty} \psi_{k} n^{k}
$$

где $\psi_{0}=1$, а $\psi_{k}$ при $k=1,2, \ldots$ дается выражением

$$
\psi_{k}=k ! \sum_{\substack{r=1,2, \ldots, k ; \nu_{r}=0,1, \ldots \\ \nu_{1}+2 \nu_{2}+\cdots+k \nu_{k}=k \\ \nu_{0}+\nu_{1}+\cdots+\nu_{k}=k}} \prod_{r=0}^{k} \frac{\alpha_{r}^{\nu_{r}}}{\nu_{r} !}
$$

в котором величины $\alpha_{k}, k=0,1,2, \ldots$, определены формулой $(2.10)$.

Соответствуюшее разложение в ряд по $u$ для дополнительного нормального модуля $\widetilde{\psi}(u)$ дается формулой

$$
\widetilde{\psi}(u)=\sum_{k=0}^{\infty} \widetilde{\psi}_{k} u^{k}
$$

в которой выражения для коэффициентов $\widetilde{\psi}_{k}$ получаются из выражений для $\psi_{k}$ заменой $\alpha_{k}$ на величины $\widetilde{\alpha}_{k}, k=0,1,2, \ldots$ (см. формулы $(2.10)$ и $(2.12)$ ).

Для уравнения состояния в дифференциальной форме получаем

$$
\begin{aligned}
& \beta \frac{d p}{d n}=\sum_{k=0}^{\infty} \psi_{k} n^{k} \quad \text { (вириальное представление) }, \\
& \beta \frac{d p}{d u}=\frac{1}{1+u} \sum_{k=0}^{\infty} \widetilde{\psi}_{k} u^{k} \quad \text { (дополнительное представление). }
\end{aligned}
$$

Почленное интегрирование ряда (2.29) приводит к вириальному разложсению уравнения состояния

$$
\beta p=\sum_{k=0}^{\infty} \frac{\psi_{k}}{k+1} n^{k+1},
$$

сравнение которого с аналогичным разложением из книги [4] показывает, что определенные в [4] неприводимые интегралы $\beta_{k}$ связаны с коэффициентами $\psi_{k}(2.27)$ соотношением

$$
\beta_{k}=\frac{1}{k} \psi_{k}
$$

а для вириальных коэффициентов получается формула

$$
B_{k}=\frac{\psi_{k}}{k+1}
$$


2.4. Представления уравнения состояния контурными интегралами. В п.5 приложения показано, что функция $\psi(n)$ (формула (2.27)) может быть также представлена контурным интегралом

$$
\psi(n)=\frac{1}{2 \pi i} \oint \frac{d \zeta}{\zeta-n \zeta \alpha(\zeta)}
$$

в котором замкнутый контур интегрирования в комплексной плоскости $\zeta$ обходит точку $\zeta_{0}$, являюшуюся корнем уравнения

$$
\alpha\left(\zeta_{0}\right)=\frac{1}{n}=v
$$

Соответствуюшее представление дополнительного нормального модуля $\widetilde{\psi}(u)(2.28)$ имеет вид

$$
\widetilde{\psi}(u)=\frac{1}{2 \pi i} \oint \frac{d \zeta}{\zeta-u \zeta \widetilde{\alpha}(\zeta)}
$$

Здесь замкнутый контур интегрирования обходит в комплексной плоскости $\zeta$ точку $\widetilde{\zeta}_{0}-$ корень уравнения

$$
\widetilde{\alpha}\left(\widetilde{\zeta}_{0}\right)=\frac{1}{u}=w .
$$

Этому представлению нормального модуля соответствует следуюшее двойственное представление уравнения состояния в дифференциальной форме при помощи контурных интегралов:

$$
\beta \frac{d p}{d n}=\frac{1}{2 \pi i} \oint \frac{d \zeta}{\zeta-n \zeta \alpha(\zeta)} \quad \text { (вириальное представление) }
$$

(замкнутый контур интегрирования обходит против часовой стрелки корень $\zeta_{0}$ уравнения (2.35));

$$
\beta \frac{d p}{d u}=\frac{1}{1+u} \frac{1}{2 \pi i} \oint \frac{d \zeta}{\zeta-u \zeta \widetilde{\alpha}(\zeta)} \quad \text { (дополнительное представление) }
$$

(замкнутый контур интегрирования обходит против часовой стрелки корень $\widetilde{\zeta}_{0}$ уравнения (2.37)).

Интегрирование по $n, u$ под знаком интеграла с учетом того, что $p \rightarrow 0$ при $n \rightarrow 0$, $u \rightarrow 0$, приводит к двойственному представлению уравнения состояния:

$$
\beta p=\frac{1}{2 \pi i} \oint \frac{d \zeta}{\alpha(\zeta)} \ln \frac{1}{1-n \alpha(\zeta)} \quad \text { (вириальное представление) }
$$

(замкнутый контур интегрирования обходит против часовой стрелки корень $\zeta=\zeta_{0}$ уравнения (2.35));

$$
\beta p=\frac{1}{2 \pi i} \oint \frac{d \zeta}{\zeta+\zeta \widetilde{\alpha}(\zeta)} \ln \frac{1+u}{1-u \widetilde{\alpha}(\zeta)} \quad \text { (дополнительное представление) }
$$


(замкнутый контур интегрирования обходит против часовой стрелки корень $\widetilde{\zeta}_{0}$ уравнения (2.37)).

В заключение данного раздела приведем еше двойственное представление удельного (отнесенного к одной частице) логарифма конфигурационного интеграла $Q_{N}[4]$

$$
\mathcal{L}=\lim _{N \rightarrow \infty} \frac{1}{N} \ln Q_{N}
$$

Имеем (см. конец п.5 приложения)

$$
\mathcal{L}(n)=\ln \frac{1}{n}-1-\frac{1}{2 \pi i} \oint \frac{d \zeta}{\zeta} \frac{1}{n \alpha(\zeta)} \ln \frac{1}{1-n \alpha(\zeta)} \quad \text { (вириальное представление) }
$$

(замкнутый контур интегрирования обходит против часовой стрелки корень $\zeta=\zeta_{0}$ уравнения (2.35));

$$
\begin{aligned}
\mathcal{L}(u)= & \ln \frac{1}{u}-1-\frac{1}{2 \pi i} \oint \frac{d \zeta}{\zeta} \frac{1}{1+\widetilde{\alpha}(\zeta)} \times \\
\times & \left(\frac{1+u}{u} \ln (1+u)-\frac{1-u \widetilde{\alpha}(\zeta)}{u} \ln (1-u \widetilde{\alpha}(\zeta))\right) \\
& \quad(\text { дополнительное представление) }
\end{aligned}
$$

(замкнутый контур интегрирования обходит против часовой стрелки корень $\widetilde{\zeta}_{0}$ уравнения (2.37)).

\section{3. ДВОЙСТВЕННОЕ НОРМАЛЬНОЕ ОПИСАНИЕ МОДЕЛЬНОГО ВЕШЕСТВА ВАН-ДЕР-ВААЛЬСА}

Под "вешеством Ван-дер-Ваальса" (вешество ВдВ) здесь понимается воображаемое "вешество", для которого уравнение Ван-дер-Ваальса [5]

$$
\left(P+\frac{a N^{2}}{V^{2}}\right)\left(V-N v_{a}\right)=N k T
$$

является точным уравнением состояния. Такое "вешество" рассматривалось в литературе. Например, в книге [6] не только анализируется поведение этого "вещества" при термодинамических циклах, но и вычисляются его термодинамические функции (энтропия и др.). Мы воспользуемся вешеством ВдВ как точно решаемой и в то же время достаточно содержательной (описываюшей фазовый переход пар-жидкость) моделью, на которой удобно проиллюстрировать введенные выше понятия.

3.1. Нормальные модули $\psi(n)$ и $\widetilde{\psi}(u)$. Пользуясь определением $(2.24)$ и вводя безразмерную обратную температуру

$$
\theta=\frac{a \beta}{v_{a}}
$$


запишем двойственное представление уравнения состояния вешества ВдВ:

$$
\begin{aligned}
& \beta p=\frac{n}{1-n}-\theta n^{2} \quad \text { (вириальное представление); } \\
& \beta p=u-\frac{\theta u^{2}}{(1+u)^{2}} \quad \text { (дополнительное представление). }
\end{aligned}
$$

Дифференцируя функцию (3.2) по $n$, функцию (3.3) по $u$, приходим к двойственному представлению уравнения состояния вещества ВдВ в дифференциальной форме:

$$
\begin{aligned}
& \beta \frac{d p}{d n}=\frac{1}{(1-n)^{2}}-2 \theta n \quad \text { (вириальное представление); } \\
& \beta \frac{d p}{d u}=1-\frac{2 \theta u}{(1+u)^{3}} \quad \text { (дополнительное представление) }
\end{aligned}
$$

Сопоставляя (3.4), (3.5) с формулами (2.25), (2.26), получаем выражения для нормальных модулей вешества ВдВ:

$$
\begin{aligned}
& \psi(n)=\frac{1}{(1-n)^{2}}-2 \theta n \quad \text { (вириальный нормальный модуль) }, \\
& \widetilde{\psi}(u)=1+u-\frac{2 \theta u}{(1+u)^{2}} \quad \text { (дополнительный нормальный модуль). }
\end{aligned}
$$

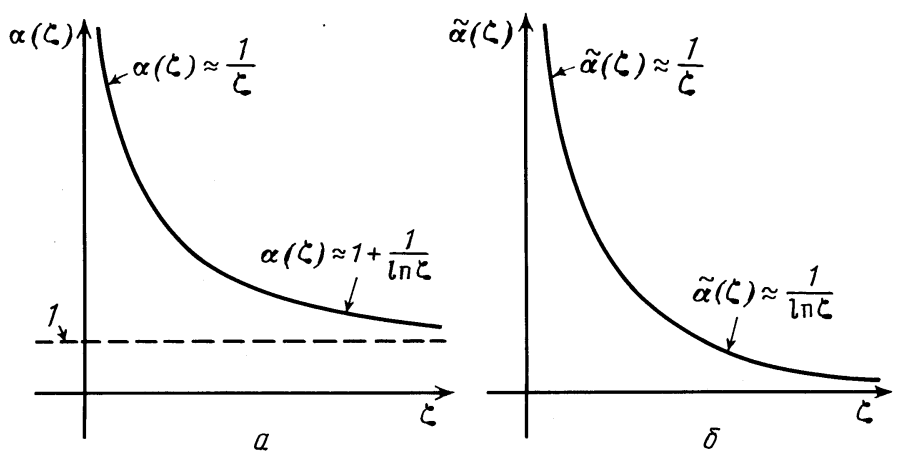

Рис. 1. Качественная картина поведения нормального объема в физической области $0<\zeta<\infty$ при температуре выше критической: $a$ - нормальный удельный объем (3.10); 6 - нормальный дополнительный объем (3.11).

3.2. Нормальная активность $\zeta$ и неявное определение нормальных объемов $v=\alpha(\zeta), w=\widetilde{\alpha}(\zeta)$. Зная нормальные модули, находим двойственное представление нормальной активности $\zeta$ (см. п.6 приложения):

$$
\zeta=\varphi(n)=\frac{n}{1-n} \exp \left(\frac{n}{1-n}-2 \theta n\right)=\phi(v)=\frac{1}{v-1} \exp \left(\frac{1}{v-1}-\frac{2 \theta}{v}\right)
$$

(вириальное представление),

$$
\zeta=\widetilde{\varphi}(u)=u \exp \left(u-\frac{2 \theta u}{1+u}\right)=\widetilde{\phi}(w)=\frac{1}{w} \exp \left(\frac{1}{w}-\frac{2 \theta}{1+w}\right)
$$

(дополнительное представление). 
Последние равенства в формулах (3.8), (3.9) задают в неявной форме функции

$$
\begin{aligned}
v=\alpha(\zeta) & \text { (вириальное представление) }, \\
w=\widetilde{\alpha}(\zeta) & \text { (дополнительное представление). }
\end{aligned}
$$

Поведение этих функций в физической области $0<\zeta<\infty$ иллюстрируется рис. 1 , из которого видно, что качественно они ведут себя в соответствии с теорией, изложенной выше в разделе 2 . Именно, при $\zeta \rightarrow 0$ в согласии с представлениями $(2.9),(2.11)$ обе имеют простой полюс $v \approx \frac{1}{\zeta}, w \approx \frac{1}{\zeta}$, а при $\zeta \rightarrow \infty$ их асимптотическое поведение согласуется с формулами (2.13), (2.14).
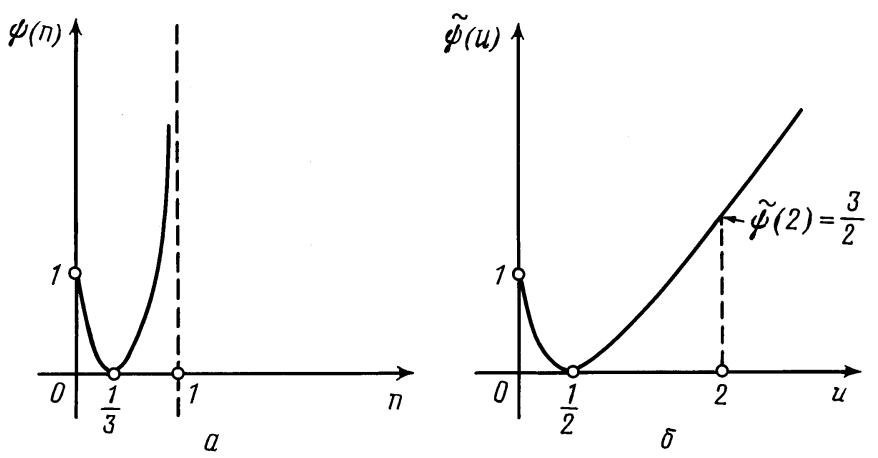

Рис. 2. Поведение нормальных модулей вещества ВдВ в физической области при температуре, равной критической: $a$ - вириальный нормальный модуль; $\sigma$ - дополнительный нормальный модуль.

3.3. Критическое поведение нормальных характеристик вещества $\mathrm{B}$ дВ. Обсудим теперь критическое поведение. Нетрудно видеть, что в критической точке вещества $\mathrm{B}_{\text {д }}{ }^{1)}$ оба его нормальных модуля обрашаются в ноль, имея при этом минимум (см. рис. 2):

$$
\begin{array}{ll}
\psi(n)=0, & d \psi / d n=0, \\
\widetilde{\psi}(u)=0, & d \widetilde{\psi} / d u=0 .
\end{array}
$$

Отсюда для критических значений имеем

$$
\begin{aligned}
& n_{c}=\frac{1}{3}, \quad \theta_{c}=\left(\frac{3}{2}\right)^{3} \quad \text { (вириальное представление) }, \\
& u_{c}=\frac{1}{2}, \quad \theta_{c}=\left(\frac{3}{2}\right)^{3} \quad \text { (дополнительное представление). }
\end{aligned}
$$

Обратим внимание, что нормальные модули $\psi(n)(3.6), \widetilde{\psi}(u)(3.7)$ разлагаются в ряды Тейлора соответственно по $n$ и $u$ в окрестностях $n=0, u=0$, причем радиусы сходимости обоих рядов Тейлора равны 1. Эти разложения по $n$ и $u$ в случае вешества ВдВ играют роль общих разложений (2.27), (2.28). Таким образом, мы приходим к важнейшему свойству нормальных модулей вещества ВдВ:

\footnotetext{
1) Определяемой обычным образом как точка перегиба в зависимости давления от объема (или плотности).
} 
ТеОремА. Для вещества ВдВ нормальные модули $\psi(n)(2.27), \widetilde{\psi}(u)(2.28)$ не имеют особенностей в критической точке (при температуре $\theta_{c}=\left(\frac{3}{2}\right)^{3}$ и значениях $\left.n=n_{c}<1, \quad u=u_{c}<1\right)$, а сама критическая точка расположсена внутри круга сходимости рядов (2.27), (2.28).

В то же время функции $v=\alpha(\zeta) \quad(3.10)$ и $w=\widetilde{\alpha}(\zeta)$ (3.11), выражающие через $\zeta$ соответственно удельный и дополняющий нормальные объемы, имеют в случае вещества ВдВ более сложное, чем нормальные модули, поведение даже в физической области. Так, нетрудно убедиться (см. п.6 приложения), что для них критическая точка является точкой ветвления, расположенной при $\theta_{c}=\left(\frac{3}{2}\right)^{3}$ и

$$
\begin{aligned}
\zeta_{c} & =\frac{1}{2 \exp (7 / 4)}, \quad v_{c}=3 \quad \text { (вириальное представление) }, \\
\zeta_{c} & =\frac{1}{2 \exp (7 / 4)}, \quad w_{c}=2 \quad \text { (дополнительное представление). }
\end{aligned}
$$

В нефизической области поведение этих функций еше сложнее.

Таким образом, из рассмотрения модельного вещества ВдВ можно сделать эвристическое заключение, что в случае систем частищ из класса систем Ван-дер-Ваальса (см. п.2.1) нормальные модули (как функции соответственно переменных $n$ (2.3) и $u$ $(2.4))$ в окрестности критической точки обладают более простым поведением, чем нормальные объемы (как функции переменной $\zeta$ ). Иными словами, рассмотрение модельного вещества ВдВ наводит на мысль, что изучение рядов Тейлора $\psi(n)(2.27), \widetilde{\psi}(u)$ (2.28) предпочтительнее изучения исходных рядов Лорана $v=\alpha(\zeta)(2.9), w=\widetilde{\alpha}(\zeta)$ $(2.11)$

3.4. Определение нормальных объемов $v, w$ вещества ВдВ в виде рядов Лорана по нормальной активности $\zeta$. Выше в формулах $(3.8),(3.9)$ функции $v=\alpha(\zeta)(3.10)$ и $w=\widetilde{\alpha}(\zeta)(3.11)$ для вешества В дВ определены в неявной форме. Выпишем здесь их явное двойственное представление рядами Лорана по $\zeta$, соответствуюшее двойственному представлению нормальных объемов $v(\zeta)(2.9), w(\zeta)(2.11)$.

Имеем (см. п.6 приложения)

$$
v=\alpha(\zeta)=\frac{1}{\zeta}+\sum_{k=1}^{\infty} \alpha_{k} \zeta^{k-1} \quad \text { (вириальное представление), }
$$

где при $k=1,2, \ldots$

и

$$
\alpha_{k}=\sum_{\substack{r=1,2, \ldots, k ; \nu_{r}=0,1, \ldots \\ \nu_{1}+2 \nu_{2}+\cdots+k \nu_{k}=k}}[-(k-1)]^{\sum_{r=1}^{k} \nu_{r}-1} \prod_{r=1}^{k} \frac{\lambda_{r}^{\nu_{r}}}{\nu_{r} !},
$$

Аналогично

$$
\lambda_{1}=2(1-\theta), \quad \lambda_{r}=\frac{r+1}{r}, \quad r=2,3, \ldots
$$

$$
w=\widetilde{\alpha}(\zeta)=\frac{1}{\zeta}+\sum_{k=1}^{\infty} \widetilde{\alpha}_{k} \zeta^{k-1} \quad \text { (дополнительное представление), }
$$

где коэффициенты $\widetilde{\alpha}_{k}, k=1,2, \ldots$, определены формулой $(3.16)$, в которой величины $\lambda_{r}$ заменены на $\widetilde{\lambda}_{r}$ :

$$
\widetilde{\lambda}_{1}=1-2 \theta, \quad \widetilde{\lambda}_{r}=2 \theta(-1)^{r}, \quad r=2,3, \ldots
$$


3.5. Представление нормальных объемов $v, w$ вешества ВдВ контурными интегралами. Имеем (см. п.6.3 приложения)

$$
\begin{gathered}
v=\alpha(\zeta)=\frac{1}{2 \pi i} \oint \frac{d \xi}{\xi} \frac{-2 \theta(1+\xi)+\frac{1+\xi}{1-y}+\frac{1}{y} \ln \frac{1}{1-y}+\frac{\xi}{(1-y)^{2}}}{\xi+2 \theta y-\frac{y}{1-y}-\ln \frac{1}{1-y}} \\
\text { (вириальное представление) },
\end{gathered}
$$

где $y=\zeta \exp (-\xi)$, а замкнутый контур интегрирования в комплексной плоскости $\zeta$ обходит против часовой стрелки корни уравнения

$$
\xi+2 \theta y-\frac{y}{1-y}-\ln \frac{1}{1-y}=0
$$

и не включает точку $\xi=0$.

Аналогично

$$
w=\widetilde{\alpha}(\zeta)=\frac{1}{2 \pi i} \oint \frac{d \xi}{\xi} \frac{1+\xi-\frac{2 \theta}{1+y}-\frac{2 \theta \xi}{(1+y)^{2}}}{\xi-y+\frac{2 \theta y}{1+y}}
$$

(дополнительное представление),

где $y=\zeta \exp (-\xi)$, а замкнутый контур интегрирования в комплексной плоскости $\zeta$ обходит против часовой стрелки корни уравнения

$$
\xi-y+\frac{2 \theta y}{1+y}=0
$$

и не включает точку $\xi=0$.

Критическое поведение контурных интегралов. При $\theta<\left(\frac{3}{2}\right)^{3}$ решение трансцендентных уравнений (3.21), (3.23) единственно. В точке $\theta=\left(\frac{3}{2}\right)^{3}$ решение этих уравнений ветвится, и при $\theta>\left(\frac{3}{2}\right)^{3}$ уравнения имеют три корня. Соответственно по три ветви имеют рассмотренные выше контурные интегралы. В п.6.3 приложения показано, что ветвление происходит при $\theta=\theta_{c}=\left(\frac{3}{2}\right)^{3}, \quad \zeta=\zeta_{c}=\frac{1}{2 \exp (7 / 4)}$ и следующих значениях $\xi=\xi_{c}$ :

$$
\begin{aligned}
& \xi_{c}=-\frac{7}{4}+\ln \frac{3}{2} \quad \text { (вириальное представление), } \\
& \xi_{c}=-\frac{7}{4} \quad \text { (дополнительное представление). }
\end{aligned}
$$

Я благодарен А.А.Сапоженко, обратившему мое внимание на работы Г.И.Калмыкова, Г.И.Калмыкову за полезные обсуждения его работ, В.С.Бескину, Б.Л.Воронову, Л.В.Келдьшу, В.В.Лосякову, М.А.Соловьеву за интерес к работе и ценные замечания, а также рецензенту, внимательно прочитавшему рукопись, помогшему устранить описки и сделать изложение более ясным.

6 Теоретическая и математическая физика, т. 108, № 1, 1996 г. 


\section{ПРИЛОЖЕНИЕ}

\section{1. ПРОБЛЕМА АСИМПТОТИЧЕСКОЙ КАТАСТРОФЫ МАЙЕРОВСКИХ РЯДОВ И ЕЕ РЕШЕНИЕ}

В работах [2] показано, что в стандартной теории майеровских разложений $[3,4]$ и ее обобщениях, получаемых на пути суммирования диаграмм по семействам гомоморфных графов [7], присутствует феномен асимптотической катастрофы, обесценивающий полученные в работах [7] строгие результаты суммирований по семействам графов.

Поясним явление асимптотической катастрофы на примере майеровских разложений по активности (см. ниже (П.6)). Коэффициенты $b_{l}$ в правых частях (П.6) выражаются майеровскими суммами по связным $l$-вершинным графам, в которых каждому связному графу с $l$ вершинами соответствует $l$-кратный интеграл по объему, занимаемому системой частиц. Число $s_{l}$ таких слагаемых равно числу связных $l$-вершинных графов. При $l \rightarrow \infty$ асимптотически (см. [8])

$$
s_{l} \approx e^{\frac{l^{2}}{2}}
$$

Поскольку все слагаемые имеют одинаковый порядок величины, результат (П.1) означает, что если бъ члены майеровской суммы не уничтожали друг друга с огромной точностью порядка

$$
C^{l} e^{-\frac{l^{2}}{2}} \quad(C-\text { константа })
$$

то ряды в правых частях (П.6) расходились бы при любом отличном от нуля.

Но эти ряды сходятся в круге ненулевого радиуса (см. $[9,10])$. Значит, при $l \rightarrow \infty$ слагаемые майеровской суммы практически полностью уничтожают друг друга. Огромное число слагаемых (П.1), растущее с ростом $l$ намного быстрее любого мыслимого закона убывания отдельных членов этой суммы, “порождает" относительно незначительный остаток порядка

$$
C^{l}(C-\text { константа })
$$

о структуре и величине которого ничего нельзя сказать на основании вида исходной майеровской суммы. Иначе говоря, при майеровском подходе приходится работать с огромным множеством не имеюших прямого отношения к делу недостаточно малых интегралов, которые должны взаимно уничтожиться, а представляющая интерес малая величина, остаюшаяся после взаимного уничтожения, не доступна непосредственному исследованию. Чтобы подчеркнуть драматизм ситуации, это свойство майеровских сумм названо в работах [2] "асимптотической катастрофой".

Тупик асимптотической катастрофы преодолен Калмыковым [1] . Он нашел представление коэффициентов $b_{l}$, общий член которого маркируется помеченным деревом $\left.{ }^{2}\right)$, а не

\footnotetext{
${ }^{2)}$ Тот факт, что любой связный граф содержит в качестве своего связного подграффа дерево, использовался, например, в работах $[11,12,13]$ для оценки сверху закона убывания многоточечных корреляций. Но, кроме цитированных статей Калмыкова [1], автору не известны работы, в которых бы явно ставился и решался вопрос о классификации связных помеченных граффов при помощи помеченных деревьев с тем же, что и в графах, числом вершин.
} 
(связным) графом обшего вида. Это сразу решает проблему. В самом деле, число помеченных деревьев $\mathrm{c} l$ вершинами равно $l^{l-2}$, а поскольку интегралы в сумме, выражающей $b_{l}$, пропорциональны $1 / l !^{3)}$, то при $l \rightarrow \infty$

$$
b_{l} \sim \frac{l^{l-2}}{l !} \simeq \frac{1}{l^{2}} e^{l} .
$$

Таким образом, коэффициент $b_{l}$ растет с ростом $l$ не быстрее, чем $C^{l}$, где $C$ - константа, в полном соответствии с оценкой (П.3), и асимптотическая катастрофа вообше не возникает: представление Калмыкова непосредственно дает тот "относительно незначительный остаток", о котором говорилось вьше.

Суть найденного Калмыковым решения проблемы асимптотической катастрофы рядов майеровского типа и, более общо, такой же катастрофы рядов теории возмушений по степеням потенциала взаимодействия можно пояснить следуюшим образом.

Майеровские разложения возникли в результате замены в исходном конфигурационном интеграле всех больимановских әкспонент $e^{-\beta \phi}$ на “функции Майера" $f$, где

$$
e^{-\beta \phi}=1+f
$$

( $\phi$ - потенциал парного взаимодействия частищ, $\left.\beta=\frac{1}{k T}\right)$. Калмыков предложил способ не тотального (как у Майера и его прямых последователей $[3,4,7]$ ), а частичного разложения по функциям Майера $f$. Он указал, как дозированно ввести майеровские функции $f$, оставляя в слагаемых сумм неразло женные части исходного конфигурационного интеграла. В древесных суммах Калмыкова, представляюших коэффишиент $b_{l}$, структура отдельных интегралов описывается связными графами специального вида, имеющими ребра двух сортов: “маейеровские” и “больцмановские”. Майеровские ребра принадлежат помеченному дереву, маркируюшему этот специальный связный граф; им в подынтегральном выражении сопоставляются сомножители $f$. Остальные ребра этого специального связного граффа больцмановские: им в подынтегральном выражении сопоставляются больцмановские экспоненты $e^{-\beta \phi}$. Знакопеременность подынтегрального выражения определяется майеровскими функциями $f$. Поскольку число ребер в любом дереве $\mathrm{c} l$ вершинами одинаково и равно $l-1$, все слагаемые древесной суммы Калмыкова, представляюшей коэффициент $b_{l}$, имеют одинаковый знак, чем обеспечивается минимизация числа слагаемых в этой сумме при заданном ее значении. Таким образом, древесные суммы Калмыкова позволяют непосредственно изучать ту представляюшую интерес малую величину, о которой говорилось выше и которая в майеровском представлении скрыта за “плотным туманом" недостаточно малых майеровских интегралов, взаимно уничтожающих друг друга.

Поскольку все слагаемые каждой древесный суммы Калмыкова имеют в ней одинаковый знак, очень легко оценивать порядок величины этих сумм. В частности, нетрудно проверить, что во всех рассмотренных в настоящей статье рядах (2.9), (2.11), (2.27)-(2.31) асимптотической катастрофы нет и эти ряды имеют ненулевой радиус сходимости в тех же областях значений параметров, в которых сходятся ряды Калмыкова [1] (мы здесь на этой проверке не останавливаемся).

\footnotetext{
3) Выше мы не учитывали этого фактора ввиду его незначительности по сравнению с основным множителем $\exp \left(l^{2} / 2\right)$.
} 
(6)

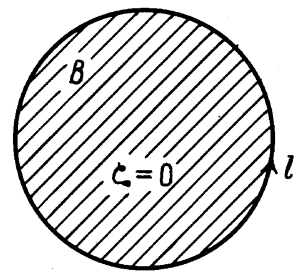

$a$

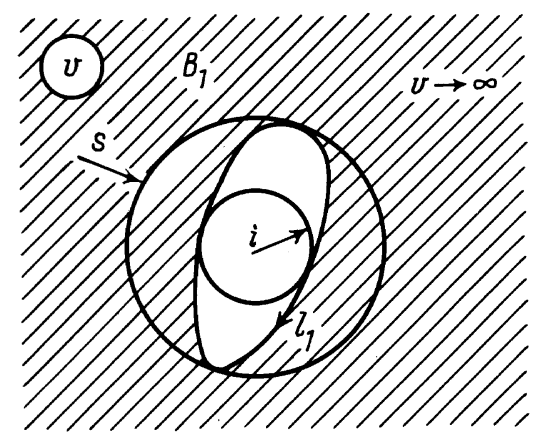

6

Рис. 3. $a$-комплексная плоскость $\zeta, 6$-комплексная плоскость $v$; $i \equiv \inf _{\zeta \in l}|\alpha(\zeta)|, s \equiv \sup _{\zeta \in l}|\alpha(\zeta)|$.

\section{2. ЗАПИСЬ УРАВНЕНИЯ СОСТОЯНИЯ В ДИФФЕРЕНЦИАЛЬНОЙ ФОРМЕ}

Из майеровских разложений [4] давления $P$ и плотности $n_{s}=\frac{N}{V}$ по активности $z$

$$
\beta P=\sum_{l=1}^{\infty} b_{l} z^{l}, \quad n_{s}=\sum_{l=1}^{\infty} l b_{l} z^{l}
$$

следует дифференциальное соотношение

$$
\beta z \frac{d P}{d z}=n_{s},
$$

которое после введения нормальной активности $\zeta(2.8)$, нормальной плотности $n(2.3)$ и давления $p(2.24)$, нормированного на объем, принимает вид

$$
\beta \zeta \frac{d p}{d \zeta}=n .
$$

С учетом определений дополняющей плотности $u(2.4)$, функций $\psi(n)(2.22)$ и $\widetilde{\psi}(u)$ (2.23) соотношение (П.7) переходит в уравнения (2.25) и (2.26).

Для вывода формулы (2.22) обратим внимание, что с учетом определений $(2.1),(2.3)$ имеем согласно (2.16), (2.18)

$$
\psi=-\frac{v}{n} \frac{d \beta p}{d v}=\frac{d \beta p}{d n}=\left(\frac{d \beta p}{d \zeta}\right)\left(\frac{d \zeta}{d n}\right),
$$

откуда и из (П.7) получаем $\psi=n \frac{d \ln \zeta}{d n}$, что с учетом определения (2.20) дает формулу (2.22). Формула (2.23) выводится аналогично.

3. ОБРАЩЕНИЕ РЯДОВ ЛОРАНА ДЛЯ ФУНКЦИЙ $v=\alpha(\zeta)$ И $w=\widetilde{\alpha}(\zeta)$

Займемся обрашением функции $v=\alpha(\zeta)(2.9)$ (обрашение дополнительной функции $w=\widetilde{\alpha}(\zeta)$ (2.11) производится аналогично). 
В комплексной плоскости $\zeta$ рассмотрим область $B$, содержащую начало координат $\zeta$ и заключенную внутри замкнутого контура $l$, обходящего начало координат против часовой стрелки в области сходимости ряда $\alpha(\zeta)(2.9)$ (см. рис. $3 a$ ). Внутри области $B$ содержится один простой полюс функции $\alpha(\zeta)$. Для такого случая проблема обрашения ряда Лорана рассмотрена, например, в книге [14]. При обходе контура $l$ по часовой стрелке значения функции $v=\alpha(\zeta)$ обходят в комплексной плоскости $v$ контур $l_{1}$ против часовой стрелки (см. рис. 36$)$.

Точка $\zeta=0$ переходит в точку $v=\infty$, и функция $v=\alpha(\zeta)$ конформно отображает область $B$, заключенную внутри контура $l$, в область $B_{1}$, внешнюю по отношению к контуру $l_{1}$. Отсюда следует, что разность $\alpha(\zeta)-v$ не имеет нуля при $|v|<\inf _{\zeta \in l}|\alpha(\zeta)|$ и имеет ровно один нуль, когда

$$
|v|>\sup _{\zeta \in l}|\alpha(\zeta)|
$$

Поэтому функция

$$
\frac{\zeta \alpha^{\prime}(\zeta)}{\alpha(\zeta)-v}
$$

не имеет полюса при $\zeta=0$ и в случае справедливости условия (П.8) заведомо имеет ровно один простой полюс при $\zeta=\phi(v)$ (при $\alpha(\zeta)=v$ ). Вычет в этом полюсе равен $\phi(v)$. По теореме Коши о вычетах

$$
\zeta=\phi(v)=\frac{1}{2 \pi i} \oint \frac{d \xi \xi \alpha^{\prime}(\xi)}{\alpha(\xi)-v},
$$

что после интегрирования по частям дает

$$
\zeta=\phi(v)=-\frac{1}{2 \pi i} \oint d \xi \ln \left(1-\frac{\alpha(\xi)}{v}\right)
$$

где логарифм понимается в смысле главного значения. Разлагая с учетом условия (П.8) логарифм в ряд и почленно интегрируя, приходим к разложению в окрестности точки $v=\infty$

$$
\zeta=\phi(v)=\sum_{k=0}^{\infty} \frac{1}{v^{k+1}} \frac{1}{k+1} \frac{1}{2 \pi i} \oint d \xi \alpha^{k+1}(\xi),
$$

которое после введения нормальной плотности $n(2.3)$ переходит в ряд

$$
\zeta=\varphi(n)=\sum_{k=0}^{\infty} \varphi_{k} n^{k+1}
$$

где

$$
\varphi_{k}=\frac{1}{k+1} \frac{1}{2 \pi i} \oint d \xi \alpha^{k+1}(\xi) .
$$

Подставляя для $\alpha(\xi)$ ряд (2.9), беря интегралы в формуле (П.10) с помощью теоремы Коши о вычетах и рассуждая по индукции, получаем для $\varphi_{k}$ выражение

$$
\varphi_{k}=k ! \sum_{\substack{r=1,2, \ldots ; \nu_{r}=0,1, \ldots \\ \nu_{1}+2 \nu_{2}+\cdots+k \nu_{k}=k \\ \nu_{0}+\nu_{1}+\cdots+\nu_{k}=k+1}} \prod_{r=0}^{k} \frac{\alpha_{r}^{\nu_{r}}}{\nu_{r} !}
$$


Здесь величины $\alpha_{r}$ определены формулой (2.9).

Повторяя изложенные рассуждения, получаем, что функция, обратная дополнительной функции $w=\widetilde{\alpha}(\zeta)(2.11)$, представляется рядом

$$
\zeta=\widetilde{\varphi}(u)=\sum_{k=0}^{\infty} \widetilde{\varphi}_{k} u^{k+1},
$$

коэффициенты которого $\widetilde{\varphi}_{k}$ даются формулой

$$
\varphi_{k}=k ! \sum_{\substack{r=1,2, \ldots, k ; \nu_{r}=0,1, \ldots \\ \nu_{1}+2 \nu_{2}+\cdots+k \nu_{k}=k \\ \nu_{0}+\nu_{1}+\cdots+\nu_{k}=k+1}} \prod_{r=0}^{k} \frac{\widetilde{\alpha}_{r}^{\nu_{r}}}{\nu_{r} !}
$$

где $\widetilde{\alpha}_{k}$ определены в формуле (2.11).

\section{4. РЯДЫ ТЕЙЛОРА ДЛЯ ФУНКЦИЙ $\psi(n)$ И $\widetilde{\psi}(u)$}

Мы построим здесь ряд для функции $\psi(n)$. Ряд для дополнительной функции $\widetilde{\psi}(u)$ строится аналогично.

Будем исходить из определения (2.22). Полагая

$$
\ln \frac{\varphi(n)}{n}=\sum_{r=1}^{\infty} \lambda_{r} n^{r},
$$

имеем

$$
\begin{aligned}
\frac{\varphi(n)}{n}=\exp \left(\sum_{r=1}^{\infty} \lambda_{r} n^{r}\right) & =\prod_{r=1}^{\infty} \exp \left(\lambda_{r} n^{r}\right)=\prod_{r=1}^{\infty} \sum_{\nu_{r}=0}^{\infty} \frac{\left(\lambda_{r} n^{r}\right)^{\nu_{r}}}{\nu_{r} !}= \\
& =\sum_{k=0}^{\infty} n^{k} \sum_{\substack{k=1,2, \ldots, k ; \nu_{r}=0,1, \ldots \\
\nu_{1}+2 \nu_{2}+\cdots+k \nu_{k}=k}}^{k} \frac{\lambda_{r} \nu_{r}}{\nu_{r} !}
\end{aligned}
$$

Сравнивая последнее выражение с формулами (П.9), (П.11), приходим к системе уравнений, связываюших величины $\lambda_{r}$ (П.14) и $\alpha_{r}(2.9)$ :

$$
\sum_{\substack{r=1,2, \ldots, k ; \nu_{r}=0,1, \ldots \\
\nu_{1}+2 \nu_{2}+\cdots+k \nu_{k}=k}} \prod_{r=1}^{k} \frac{\lambda_{r}{ }^{\nu_{r}}}{\nu_{r} !}=k ! \sum_{\begin{array}{c}
r=1,2, \ldots, k ; \nu_{r}=0,1, \ldots \\
\nu_{1}+2 \nu_{2}+\cdots+k \nu_{k}=k \\
\nu_{0}+\nu_{1}+\cdots+\nu_{k}=k+1
\end{array}} \prod_{r=0}^{k} \frac{\alpha_{r}^{\nu_{r}}}{\nu_{r} !}
$$

Эта система уравнений решается по индукции, решение имеет вид

$$
\lambda_{k}=(k-1) ! \sum_{\substack{r=1,2, \ldots, k ; \nu_{r}=0,1, \ldots \\ \nu_{1}+2 \nu_{2}+\cdots+k \nu_{k}=k \\ \nu_{0}+\nu_{1}+\cdots+\nu_{k}=k}} \prod_{r=0}^{k} \frac{\alpha_{r}^{\nu_{r}}}{\nu_{r} !} .
$$


Подставляя формулы (П.14) и (П.16) в определение $\psi(n)(2.22)$, выполняя почленное дифференцирование и учитывая определения $(2.9),(2.10)$, получаем для разложения вириального нормального модуля $\psi(n)$ в рядпо нормальной плотности $n$ формулу $(2.27)$.

Совершенно аналогичные рассуждения показывают, что разложение дополнительного нормального модуля $\widetilde{\psi}(u)$ в ряд по дополняющей плотности $u$ дается формулой (2.28).

\section{5. КОНТУРНЫЕ ИНТЕГРАЛЫ ДЛЯ ФУНКЦИЙ $\psi(n)$ И $\widetilde{\psi}(u)$}

Сначала получим контурные интегралы для вириального нормального модуля $\psi(n)$. Соответствуюшие представления дополнительного нормального модуля строятся аналогично.

Непосредственная проверка с применением теоремы Коши о вычетах показывает, что ряд (2.27) может быть записан в виде

$$
\psi(n)=\sum_{k=0}^{\infty} u^{k} k ! \frac{1}{2 \pi i} \oint \frac{d \xi}{\xi^{k+1}} \frac{1}{2 \pi i} \oint \frac{d \zeta}{\zeta^{k+1}} \exp (\xi \zeta \alpha(\zeta)) .
$$

Пользуясь представлением $k !=\int_{0}^{\infty} d t e^{-t} t^{k}$ и изменяя порядок суммирования и интегрирования, приводим предыдуший ряд к виду

$$
\psi(n)=\int_{0}^{\infty} d t e^{-t} \frac{1}{2 \pi i} \oint \frac{d \xi}{\xi} \frac{1}{2 \pi i} \oint \frac{d \zeta}{\zeta} \exp (\xi \zeta \alpha(\zeta)) \sum_{k=0}^{\infty}\left(\frac{n t}{\xi \zeta}\right)^{k}
$$

При каждом $t$ выбираем $\xi$ так, чтобы было $\left|\frac{n t}{\zeta}\right|<|\xi|$. При таком выборе ряд для геометрической прогрессии в (П.17) сходится, а точка

$$
\xi_{0}=\frac{n t}{\zeta}
$$

находится внутри замкнутого контура, охватывающего начало координат в комплексной плоскости $\xi$. Суммируя геометрическую прогрессию в (П.17) и изменяя порядок интегрирования по $\xi$ и $\zeta$, получаем

$$
\psi(n)=\int_{0}^{\infty} d t e^{-t} \frac{1}{2 \pi i} \oint \frac{d \zeta}{\zeta} \frac{1}{2 \pi i} \oint \frac{d \xi}{\xi-\frac{n t}{\zeta}} \exp (\xi \zeta \alpha(\zeta))
$$

после чего интеграл по $\xi$ берется как простой вычет в точке $\xi_{0}$ (П.18) и

$$
\psi(n)=\int_{0}^{\infty} d t e^{-t} \frac{1}{2 \pi i} \oint \frac{d \zeta}{\zeta} \exp (n t \alpha(\zeta))
$$




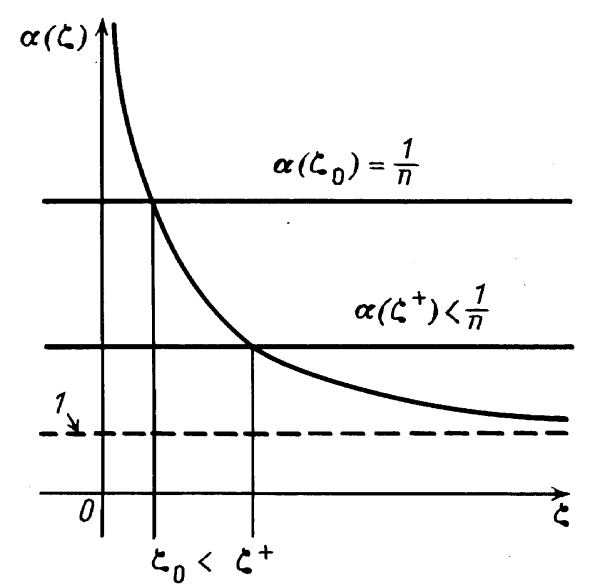

Рис. 4. Относительное положение корня $\zeta_{0}$ уравнения (П.21) и координаты $\zeta^{+}$точки пересечения контура интегрирования с вещественной осью $\zeta$.

Замкнутый контур интегрирования в комплексной плоскости $\zeta$ по определению обходит против часовой стрелки начало координат и расположен внутри круга сходимости ряда $\alpha(\zeta)$ (2.9). Вибираем этот контур так, чтобы на нем удовлетворялось условие

$$
\sup \operatorname{Re} n \alpha(\zeta)<1,
$$

которое заведомо выполнено при $n \rightarrow 0$. Напомним, что для ван-дер-ваальсовых систем по определению (см. формулы (2.9), (2.13)) $\alpha(\zeta) \rightarrow+\infty$ при $\zeta \rightarrow 0^{+}$и $\alpha(\zeta) \rightarrow+1$ при $\zeta \rightarrow+\infty$. Поэтому для этих систем частиц функция $\alpha(\zeta)$ является в целом убывающей. Она монотонно убывает при достаточно малых $n$ и $\beta$ (2.17) (при температурах выше критической), а при температурах ниже критической эта функция многолистна и на вешественной оси $\zeta$ содержит область неоднозначности при некоторых промежуточных $\zeta$, $0<\zeta<+\infty^{4)}$. Отсюда следует, что условие (П.20) означает такой выбор контура интегрирования в комплексной плоскости $\zeta$, при котором корень уравнения

$$
\alpha\left(\zeta_{0}\right)=\frac{1}{n}=v
$$

заключен внутри контура. Расположение контура интегрирования пояснено на рис. 4 , а графическое решение уравнения (П.21) - на рис. 5. При условии (П.20) в правой части (П.19) можно изменить порядок интегрирования и взять интеграл по $t$, что дает

$$
\psi(n)=\frac{1}{2 \pi i} \oint \frac{d \zeta}{\zeta-n \zeta \alpha(\zeta)},
$$

где по определению замкнутый контур интегрирования охватывает точки $\zeta=0$ и $\zeta=\zeta_{0}$ (П.21). Но поскольку

$$
\lim _{\zeta=0} \zeta \alpha(\zeta)=1
$$

\footnotetext{
${ }^{4)}$ Подробнее об этом см. раздел 3 и п.6 приложения.
} 

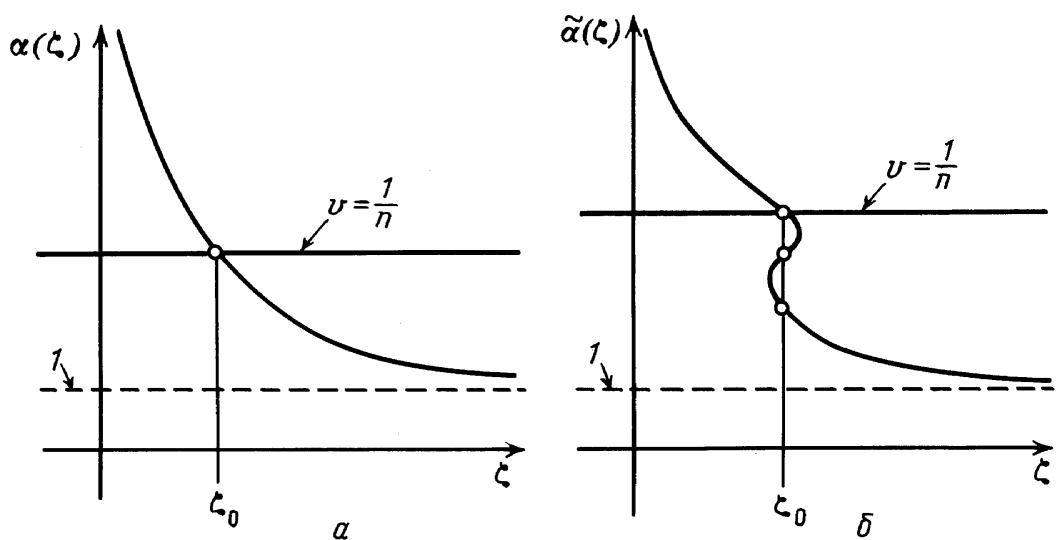

Рис. 5. Графическая иллюстрация к решению уравнения (П.21): $a$ - температура выше критической; 6 - температура ниже критической.

подынтегральное выражение (П.22) не имеет особенностей при $\zeta=0$ и замкнутый контур интегрирования в (П.22) можно сместить так, чтобы он охватьвал только корень $\zeta_{0}$ уравнения (П.21). Тогда мы приходим к выражению (2.34) раздела 2.

Нетрудно убедиться, что из интегрального представления (П.22) непосредственно следует исходное определение $\psi(n)$ (2.22), что является прямым доказательством законности всех промежуточных выкладок, приводящих к формуле (П.22). Покажем это в простейшем случае высоких температур (низких плотностей), когда уравнение (П.21) имеет всего один корень $\zeta=\zeta_{0}$. Разлагая знаменатель подынтегрального выражения (П.22) в ряд Тейлора в окрестности точки $\zeta=\zeta_{0}$, учитывая, что $n \alpha\left(\zeta_{0}\right)=1$, и беря простой вычет, приводим правую часть (П.22) к виду

$$
-\frac{1}{n} \frac{1}{\zeta_{0}} \frac{d \zeta_{0}}{d \alpha}
$$

Но согласно формуле (2.9) $\alpha=v=\frac{1}{n}$. Поэтому выражение (П.23) переходит в

$$
n \frac{1}{\zeta} \frac{d \zeta}{d n}
$$

что совпадает с правой частью определения (2.22).

Совершенно аналогичная аргументация приводит для дополнительного нормального модуля $\widetilde{\psi}(u)$ к интегральному представлению $(2.36)$, которое равносильно исходному определению (2.23).

Остановимся еше на двойственном представлении удельного (отнесенного к одной частице) логарифма конфигурационного интеграла $Q_{N}$ (см. конец раздела 2, п.4). Вывод дадим для вириального представления (случай дополнительного представления рассматривается аналогично). Термодинамическое соотношение

$$
P=-\left(\frac{\partial F}{\partial V}\right)_{T}=k T\left(\frac{\partial Q_{N}}{\partial V}\right)_{T}
$$


( $F$ - свободная энергия) с учетом определения $p$ (см. (2.24)) записывается в виде

$$
\beta p=-n^{2} \frac{\partial \mathcal{L}}{\partial n}
$$

откуда

$$
\mathcal{L}=-\beta \int \frac{d n}{n^{2}} p(n)
$$

Непосредственная подстановка сюда выражения (2.40) приводит к расходимости интеграла на нижнем пределе (при $n=0)$. Это затруднение, однако, тривиально обходится путем добавления и вычитания выражения $\mathcal{L}=\mathcal{L}_{\text {ид }}$ для идеального газа

$$
\mathcal{L}_{\text {ид }}=\ln v=\ln \frac{1}{n}=-\int \frac{d n}{n},
$$

после чего интеграл становится сходящимся и формула (2.42) получается немедленно.

\section{6. НОРМАЛЬНЫЕ ХАРАКТЕРИСТИКИ ВЕЩЕСТВА ВАН-ДЕР-ВААЛЬСА}

6.1. Нормальная активность $\zeta$. Построим для вешества ВдВ функции

$$
\begin{array}{rlrl}
\zeta & =\varphi(n)=\phi(v), & v=\alpha(\zeta) \quad \text { (вириальное представление) }, \\
\zeta=\widetilde{\varphi}(u)=\widetilde{\phi}(w), \quad w=\widetilde{\alpha}(\zeta) \quad \text { (дополнительное представление). }
\end{array}
$$

Конкретные рассуждения дадим для вириального представления. Случай дополнительного представления рассматривается аналогично.

Записывая левую часть уравнения (3.4) в форме $\beta \zeta \frac{d p}{d \zeta} \frac{1}{\zeta} \frac{d \zeta}{d n}$ и заменяя согласно (П.7), комбинацию $\beta \zeta \frac{d p}{d \zeta}$ на $n$, приходим к дифференциальному уравнению

$$
\frac{n}{\zeta} \frac{d \zeta}{d n}=\frac{1}{(1-n)^{2}}-2 \theta n,
$$

решая которое при условии $\lim _{n \rightarrow 0} \frac{\zeta}{n}=1$, получаем представление (3.8).

6.2. Ряды Лорана по $\zeta$ для нормальных удельного и дополнительного объемов $v=\alpha(\zeta), w=\widetilde{\alpha}(\zeta)$. Формулы $(3.8)$ дают представление функций $v=\alpha(\zeta)$ $(3.10)$ и $w=\widetilde{\alpha}(\zeta)(3.11)$ для вешества ВдВ в неявной форме. Переходя к их явному аналитическому определению, начнем с представления рядами Лорана. Вывод приведем для функции

$$
v=\alpha(\zeta)=\frac{1}{\zeta}+\sum_{k=1}^{\infty} \alpha_{k} \zeta^{k-1}
$$

Случай $w=\widetilde{\alpha}(\zeta)$ рассматривается аналогично.

Записывая формулу (3.8) в виде

$$
\zeta=n \exp \left(\sum_{k=1}^{\infty} \lambda_{k} n^{k}\right)
$$


где $\lambda_{1}=2(1-\theta), \lambda_{k}=\frac{k+1}{k}, k=2,3, \ldots$, выражая экспоненту бесконечным произведением и разлагая его сомножители в ряд, приходим к представлению

$$
\zeta=\sum_{k=0}^{\infty} n^{k+1} \sum_{\substack{r=1,2, \ldots, k ; \nu_{r}=0,1, \ldots \\ \nu_{1}+2 \nu_{2}+\cdots+k \nu_{k}=k}} \prod_{r=1}^{k} \frac{\lambda_{r}^{\nu_{r}}}{\nu_{r} !}
$$

Сравнивая последнюю формулу с разложением (П.9), (П.11), приходим к системе уравнений, связьваюшей коэффициенты $\alpha_{k}$ с коэффициентами $\lambda_{k}$ и по форме совпадаюшей с системой (П.15). Решая ее относительно $\alpha_{k}$ по индукции, выражаем коэффициенты $\alpha_{k}$ через коэффициенты $\lambda_{k}$ :

$$
\alpha_{k}=\sum_{\substack{r=1,2, \ldots, k ; \nu_{r}=0,1, \ldots \\ \nu_{1}+2 \nu_{2}+\cdots+k \nu_{k}=k}}[-(k-1)]^{\sum_{r=1}^{k} \nu_{r}-1} \prod_{r=1}^{k} \frac{\lambda_{r}^{\nu_{r}}}{\nu_{r} !} .
$$

Формулы (П.24), (П.26) равносильны уравнениям (3.15)-(3.17).

6.3. Контурные интегралы для нормальных объемов $v=\alpha(\zeta), w=$ $\widetilde{\alpha}(\zeta)$. Перейдем теперь к представлению функций $v=\alpha(\zeta) \quad(3.10)$ и $w=\widetilde{\alpha}(\zeta) \quad(3.11)$ для вешества ВдВ однократными контурными интегралами. Вывод дадим для функции $v=\alpha(\zeta)$. Случай $w=\widetilde{\alpha}(\zeta)$ рассматривается аналогично.

Непосредственно проверяется, что

$$
[-(k-1)]^{\sum_{r=1}^{k} \nu_{r}-1}=\int_{0}^{\infty} d t e^{-t} t^{\sum_{r=1}^{k} \nu_{r}-1} \frac{1}{2 \pi i} \oint \frac{d \xi}{\xi^{\sum_{r=1}^{k} \nu_{r}}} e^{-(k-1) \xi},
$$

где контур интегрирования обходит против часовой стрелки начало координат в комплексной плоскости $\xi$. Подставляя это выражение в формулы (П.24), (П.26) и принимая во внимание определение $\lambda$ (П.25), имеем

$$
\begin{aligned}
\alpha(\zeta)= & \frac{1}{\zeta}+\frac{1}{\zeta} \int_{0}^{\infty} d t \frac{e^{-t}}{t} \frac{1}{2 \pi i} \oint d \xi e^{\xi}\left\{\exp \left(\frac{t}{\xi} \sum_{r=1}^{\infty} \lambda_{r}\left(\zeta e^{-\xi}\right)^{r}\right)-1\right\}= \\
= & \frac{1}{\zeta}+\frac{1}{\zeta} \int_{0}^{\infty} d t \frac{e^{-t}}{t} \frac{1}{2 \pi i} \oint d \xi e^{\xi} \times \\
& \times\left\{\exp \left[\frac{t}{\xi}\left(-2 \theta \zeta e^{-\xi}+\frac{\zeta e^{-\xi}}{1-\zeta e^{-\xi}}+\ln \frac{1}{1-\zeta e^{-\xi}}\right)\right]-1\right\} .
\end{aligned}
$$

Логарифм в показателе экспоненты можно рассматривать в смысле его главного значения при условии, что на контуре интегрирования переменная $\xi$ удовлетворяет условию

$$
\operatorname{Re} \xi>\ln \zeta
$$

Тогда на контуре функция в показателе экспоненты в фигурных скобках однозначна и после интегрирования по $\xi$ по частям имеем

$$
\begin{aligned}
& \alpha(\zeta)=\frac{1}{\zeta}+\int_{0}^{\infty} d t \frac{e^{-t}}{t} \frac{1}{2 \pi i} \oint \frac{d \xi}{\xi^{2}} \\
& \times \exp \left[\frac{t}{\xi}\left(-2 \theta y+\frac{y}{1-y}+\ln \frac{1}{1-y}\right)\right] \\
&
\end{aligned}
$$


где $y=\zeta e^{-\xi}$. Условие сходимости интеграла по $t$ в правой части (П.28)

$$
\operatorname{Re}\left\{-1+\frac{1}{\xi}\left[-2 \theta y+\frac{y}{1-y}+\ln \frac{1}{1-y}\right]\right\}<0
$$

равносильно требованию, чтобы корень $\xi_{0}$ уравнения

$$
-1+\frac{1}{\xi}\left[-2 \theta y+\frac{y}{1-y}+\ln \frac{1}{1-y}\right]=0
$$

был расположен внутри замкнутого контура интегрирования в комплексной плоскости $\xi$. Выполняя интегрирование по $t$, приходим к формуле

$$
\alpha(\zeta)=\frac{1}{\zeta}+\frac{1}{2 \pi i} \oint \frac{d \xi}{\xi} \frac{-2 \theta(1+\xi)+\frac{1+\xi}{1-y}+\frac{1}{y} \ln \frac{1}{1-y}+\frac{\xi}{(1-y)^{2}}}{\xi+2 \theta y-\frac{y}{1-y}-\ln \frac{1}{1-y}}
$$

в которой замкнутый контур интегрирования охватывает точки $\xi=0$ и $\xi=\xi_{0}$, где $\xi_{0}-$ корень уравнения (П.30). Вычет в простом полюсе $\xi=0$ равен $-\frac{1}{\zeta}$ : его вклад взаимно уничтожается с первым слагаемым (П.31). В итоге приходим к формулам (3.20), (3.21).

Формулы (3.22), (3.23) для дополнительного случая выводятся аналогично.

Критическое поведение контурных интегралов, выражающих функции $v=\alpha(\zeta), w=\widetilde{\alpha}(\zeta)$. Значения интегралов (3.20), (3.22) определяются вычетами в точках $\xi$, являюшихся соответственно корнями трансцендентных уравнений (3.21) и (3.23). Очевидно, что при $\theta \rightarrow 0$ каждое из этих уравнений имеет один вешественный корень. При увеличении $\theta$ достигается значение $\theta=\theta_{c}$, при котором происходит ветвление решения этих трансцендентных уравнений, так что при $\theta>\theta_{c}$ уравнения имеют по три вещественных корня $\xi$.

Продемонстрируем это на примере уравнения (3.21). Случай дополнительного трансцендентного уравнения (3.23) рассматривается аналогично.

Обозначим $f_{1}(\xi)=\xi, f_{2}(\xi)=-2 \theta y+\frac{y}{1-y}+\ln \frac{1}{1-y}$, где $y=\zeta e^{-\xi}$. Очевидно, что корень уравнения (3.21) есть координата пересечения графиков функций $f_{1}(\xi)$ и $f_{2}(\xi)$. Несложный графический анализ показывает, что ветвление решения трансцендентного уравнения (3.21) наступает тогда, когда точка перегиба функции $f_{2}(\xi)$ инцидентна прямой $f_{1}(\xi)$ и одновременно кривая $f_{2}(\xi)$ в точке перегиба касается прямой $f_{1}(\xi)$ (см. рис 6). Условие инцидентности записывается в виде двух соотношений $f_{1}(\xi)=f_{2}(\xi), \frac{d^{2} f_{2}(\xi)}{d \xi^{2}}=0$, а условие касания - в виде соотношения $1=\frac{d f_{2}(\xi)}{d \xi}$. Подставляя выражения $f_{1}(\xi), f_{2}(\xi)$ и учитывая, что по определению (П.28) $\frac{d y}{d \xi}=-y$, получаем из условия $\frac{d^{2} f_{2}(\xi)}{d \xi^{2}}=0$ соотношение

$$
\theta=\frac{1}{1-y}
$$

подставляя которое в условие касания, находим

$$
y=\frac{1}{3}, \quad \theta=\left(\frac{3}{2}\right)^{3} .
$$




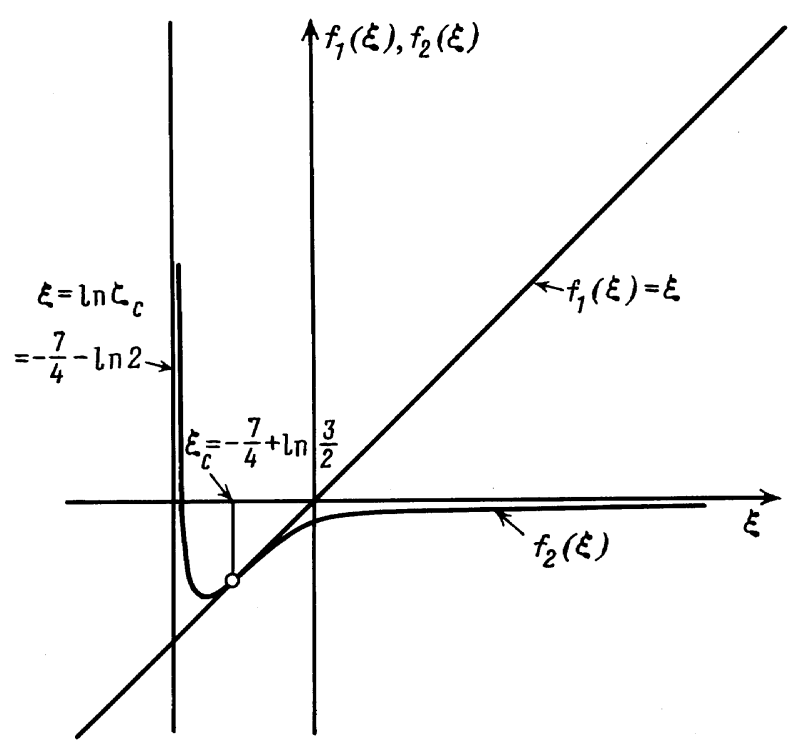

Рис. 6. Граффическое решение уравнения (3.21) в критической точке. Асимптота $\xi=\ln \zeta$ ограничивает область значений $\xi$, удовлетворяющих условию (П.27).

Из этих равенств и из условия инцидентности имеем

$$
\xi=-\frac{7}{4}+\ln \frac{3}{2}
$$

Учитывая определение $y$ (П.28), получаем

$$
\zeta=\frac{1}{2 \exp (7 / 4)}
$$

Тем самым формула (3.24) для вириального случая доказана.

Для дополнительного случая эта формула доказывается аналогично.

Работа поддержана Российским фондом фундаментальных исследований, проект № 95-02-04944-a.

\section{Список литературы}

[1] Г.И.Калмыков // ТМФ. 1990. Т. 84. №2. С. 279-289; 1992. Т. 92. №1. С. 139-149; // Дискретная математика. 1992. Т. 4. № 2. С. 66-73.

[2] И.И.Иванчик. Ковариантный метод суммирования диаграмм в классической статистической механике: Дис. докт. физ.-матем. наук. М.: Физический институт имени П.Н.Лебедева АН CCCP, 1987; I.I.Ivanchik. Generalized Mayer Series in Classical Statistical Mechanics. New York: Nova Science Publishers, Inc., 1993.

[3] Дж.Майер, М.Гепперт-Майер. Статистическая механика. М.: Мир, 1980.

[4] А.Исихара. Статистическая физика. М.: Мир, 1973.

[5] J.D.Van der Waals. Doctoral Dissertation. University of Leiden, 1873. 
[6] Энрико Ферми. Термодинамика. Пер. с англ. Харьков: Изд-во Харьковского университета, 1969.

[7] T.Morita // Progr. Theor. Phys. 1958. V. 20. №6. P. 920-938; 1959. V. 21. № 3. P. 361-382; 1960. V. 23. № 1. P. 175-177; № 5. P. 829-845; J.M.J. van Leeuwen, J.Groeneveld, J. de Boer // Physica. 1959. V. 25. №9. P. 792-808; T.Morita, K.Hiroike // Progr. Theor. Phys. 1960. V. 23. №6. P. 1003-1027; C. de Dominicis // J. Math. Phys. 1962. V. 3. №5. P. 983-1002; А.Н.Васильев. Функциональные методы в квантовой теории поля и статистике. Л.: Изд. ЛГУ, 1976; Г.А.Мартынов // ДАН СССР. 1974. Т. 218. №4. С. 814-817; И.И.Иванчик // ДАН СССР. 1987. Т. 296. №2. С. 341-344; 1988. Т. 300. №3. С. 596-600.

[8] Ф.Харари, Е.М.Палмер. Перечисления графов. М.: Мир, 1977.

[9] O.Penrose // J. Math. Phys. 1963. V. 4. № 10. P. 1312-1319.

[10] Д.Рюәль. Статистическая механика. Строгие результаты. Пер. с англ. под ред. Р.А.Минлоса. М.: Мир, 1971.

[11] М.Дюно, Б.Суйар // В сб.: Гиббсовские состояния в статистической физике. Пер. с англ. под ред. Р.А.Минлоса. М.: Мир, 1978. С. 89-106.

[12] М.Дюно, Б.Суйар, Д.Яголницер // В сб.: Гиббсовские состояния в статистической физике. Пер. с англ. под ред. Р.А.Минлоса. М.: Мир, 1978. С. 107-121.

[13] В.А.Малышев, Р.А.Минлос. Гиббсовские случайные поля. М.: Наука, 1985.

[14] В.И.Смирнов. Курс высшей математики. Т. ІІІ. Ч. 2. М.-Л.: ГИТТЛ, 1951.

Физический институт им. П.Н.Лебедева РАН

Поступила в редакцию

Центр физических исследований им. П.Н.Лебедева

6.VII.1995 г.

\section{I. Ivanchik}

\section{ANALYTIC REPRESENTATION FOR THE EQUATION OF STATE IN CLASSICAL STATISTICAL MECHANICS}

For the system of classical particles interacting by pair short-range forces the explicitly convergent expansions on usual and complementary density and the contour integral representations are constructed for the all-round compression modulus, the modulus being sufficient to describe all the thermodynamic properties and being tentatively single-valued in the vicinity of the phase transition point. With the help of those the analytic representations of the equation of state as well as specific configuration integral are found. The elaborated technique is approved on the exactly solved model - the theory of the Van der Waals substance being a model "substance" for which the Van der Waals equation is the exact equation of state. 